\section{Stable Prenucleation Calcium Carbonate Clusters}

\author{
Denis Gebauer, Antje Völkel, Helmut Cölfen ${ }^{\star}$
}

Calcium carbonate forms scales, geological deposits, biominerals, and ocean sediments. Huge amounts of carbon dioxide are retained as carbonate ions, and calcium ions represent a major contribution to water hardness. Despite its relevance, little is known about the precipitation mechanism of calcium carbonate, and specified complex crystal structures challenge the classical view on nucleation considering the formation of metastable ion clusters. We demonstrate that dissolved calcium carbonate in fact contains stable prenucleation ion clusters forming even in undersaturated solution. The cluster formation can be characterized by means of equilibrium thermodynamics, applying a multiple-binding model, which allows for structural preformation. Stable clusters are the relevant species in calcium carbonate nucleation. Such mechanisms may also be important for the crystallization of other minerals.

$\mathrm{C}$ alcium carbonate has great scientific relevance in biomineralization and geosciences, forming enormous scales of biological (reefs and ocean sediments) and geological origin, which bind a huge amount of $\mathrm{CO}_{2}$ and affect the chemistry of ocean water $(I)$ and, with it, Earth's atmosphere and climate. Scale formation (incrustation) also affects daily life, industry, and technology and can require the addition of scale inhibitors to laundry detergents and household cleaners and in many industrial applications. Scale formation also lowers the efficiency of heating and cooling devices and can result in machine damage. $\mathrm{CaCO}_{3}$ provides a model system for nucleation and crystallization analysis of minerals for classical (2) and nonclassical crystallization (3) and has been studied for more than a century. Nevertheless, little is known about the very early stages of its crystallization, that is, the prenucleation stage. Amorphous calcium carbonate (ACC) is identified as a postnucleation-stage precursor phase in calcium

Max Planck Institute of Colloids and Interfaces, Research Campus Golm, Am Mühlenberg 1, D-14424 Potsdam, Germany.

*To whom correspondence should be addressed. E-mail: coelfen@mpikg.mpg.de carbonate mineralization $(4,5)$, in bio - $(6)$ and biomimetic mineralization $(7)$, and liquid precursors have been identified in some cases (8). There is growing evidence that different species of ACC exist, that is, where the amorphous phase shows a specific short-range order that corresponds to the long-range order of the particular crystalline polymorph $(9-11)$. Besides stable biogenic species, ACC occurs as a transient precursor phase in biomineralization. Precursor species that form still earlier than ACC or liquid precursors - that is, directly after ion contact and before nucleation occurs - have been postulated (12) and suggested through modeling approaches (I3).

In the classical picture, nucleation is considered to take place in a solution of ions that has become supersaturated, leading to the nucleation of the solid phase by stochastic solute clustering, and the earliest crystal precursor is considered to be a cluster of critical size $(14,15)$. Because of the stochastic formation mechanism, such metastable clusters are a rare species. In contrast, there is increasing evidence that small polymeric species and stable clusters play a dominant role in the prenucleation stage of biomineralization and the formation of organic nanoparticles $(16,17)$.
Such soluble species have been reported for the polycondensation of silicic acid (18), precipitation of aluminum oxyhydroxide (19), and aqueous solutions of hydrated ions of the transition metals iron, chromium, uranium, molybdenum, and tungsten (20). In the above examples, the prenucleation cluster formation is a polymerizationlike event, because the chemical bonds formed are mostly covalent (silica) to partly ionic (transition metals). For nonpolymerizing ionic crystals, solute clustering has been reported only for highly soluble compounds such as citric acid, urea, sodium nitrate, and potassium sulfate (2l) and in supersaturated solutions (22). Cluster formation for low concentrations, that is, undersaturated and slightly supersaturated ionic solutions, has not been reported, and even advanced data analysis like induction time statistics do not allow for the accurate observation of all subcritical species present in a dilute system $(23,24)$.

Our experiments are based on the measurement of $\mathrm{Ca}^{2+}$ concentrations at constant $\mathrm{pH}$ values, facilitating a quantitative determination of all species present at the different stages of crystallization while the supersaturation slowly evolves. This is achieved by slow addition of dilute calcium chloride solution into dilute carbonate buffer to induce supersaturation, causing nucleation and precipitation of calcium carbonate. The experimental set-up is described in detail in supporting online material (SOM) section 1 (fig. Sl). The increase in calcium ions is shown for a single experiment at $\mathrm{pH}=9.25$ (Fig. 1A). The red line reflects the amount of calcium ions added. However, the amount of free calcium ions detected by the calcium ion selective electrode (black line) increases considerably slower straight from the beginning of the experiment; that is, a distinct part of free calcium ions disappears due to binding. The prenucleation-stage time development is linear, indicating that the calcium binding behavior in under- and supersaturated stages of the prenucleation stage is equal. Once a critical point is reached, nucleation occurs and the amount of free calcium ions drops 
to a value that corresponds to the particular solubility concentration of the precipitated phase according to a typical LaMer diagram (25).

Measurements of the time development of the amount of free calcium ions for five $\mathrm{pH}$ levels within an interval from 9.00 to 10.0 show good reproducibility (fig. $\mathrm{S} 2$ ). The $\mathrm{pH}$ range investigated is rather limited because the fraction of carbonate ions in the buffer is very low below a $\mathrm{pH}$ of 9.00 and hinders precipitation of calcium carbonate, while concurrent precipitation of calcium hydroxide occurs at $\mathrm{pH}$ values that are too high. The differences between the averages of measured free amounts and the dosed amount of calcium ions give the averaged amount of bound calcium ions (Fig. 1B). The dotted red line represents $100 \%$ binding of dosed calcium ions. Both in the prenucleation stage (i.e., before the sigmoid increase of the amount of bound calcium) and in the postnucleation stage, an increasing tendency for calcium binding with increasing $\mathrm{pH}$ is observed. The increasing binding tendency is based on the higher fraction of carbonate ions in the carbonate buffer at higher pH (SOM section 2.1 and fig. S3). Solid calcium carbonate, like any solid, has an activity of 1 by definition. Thus, the dissolution equilibrium is characterized by the ion product of calcium and carbonate ions, giving the solubility constant. To maintain the solubility constant, which, however, slightly differs for the phases precipitated in different $\mathrm{pH}$ ranges, the solubility concentration of calcium ions is specifically lower at higher concentrations of carbonate (higher $\mathrm{pH}$ ). In this way, the binding of calcium ions in solid calcium carbonate increases with increasing $\mathrm{pH}$. Similar binding of calcium ions is observed in the prenucleation stage. About $35 \%$ of added calcium ions are bound at $\mathrm{pH}=9.00$ ( $\sim 4 \%$ carbonate ions in the buffer equilibrium), whereas $\sim 75 \%$ of added calcium ions are bound at $\mathrm{pH}=10.0(-25 \%$ carbonate ions in the buffer equilibrium). This shows that calcium binding during the prenucleation stage depends on the carbonate concentration, that is, calcium carbonate clusters form. It is important to note that these clusters form in both the undersaturated and supersaturated stages (Fig. 1A). An activity of 1 cannot be assigned to clusters, because they have to be considered as solutes. Basically, this is the reason for the linear increase in free calcium ions during the prenucleation stage in contrast to the postnucleation stage (Fig. IA). After nucleation, further added calcium is consumed by the growth of particles of constant activity, giving rise to a constant solubility concentration. In the prenucleation stage, further added calcium ions are consumed by the increasing activity of clusters. The increasing fraction of carbonate ions in
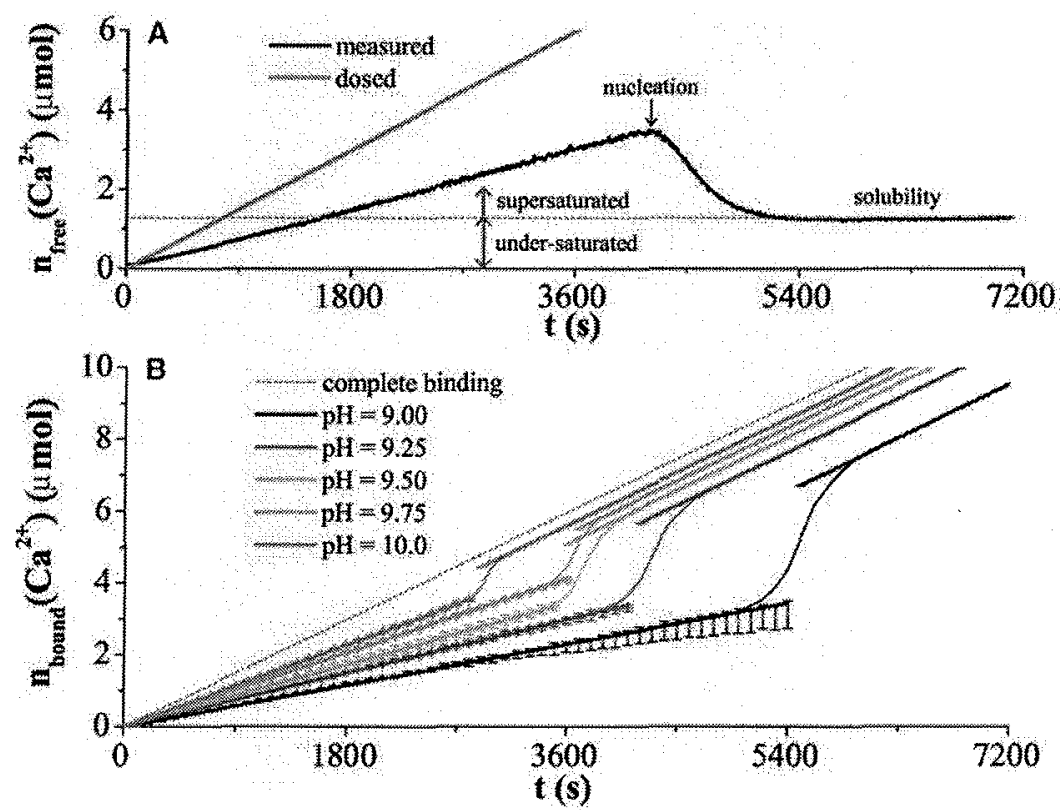

Fig. 1. (A) Development of the free calcium ions measured by the calcium ion selective electrode (black line) at $\mathrm{pH}=9.25$ in comparison with the dosed amount of calcium ions (red line). The solubility concentration of the precipitated phase defines the undersaturated and supersaturated stages of the prenucleation stage. Already in undersaturated solution, calcium ions are bound. (B) Averaged amount of bound calcium ions as calculated from the difference between the measured amount of free calcium ions and the dosed amount of calcium ions shown in $(A)$ for five pH levels. The arithmetic average of a sample of three measurements is calculated in the prenucleation stage and the postnucleation stage. Averaging during nucleation is not appropriate, and the particular developments are indicated by fine lines. Error bars depict \pm 1 SD of a sample of three measurements; the center of the error bars gives the arithmetic average of the particular data points (see also fig. $\$ 2$ ).

the buffer at higher $\mathrm{pH}$ promotes cluster formation and in this way increases calcium binding (principle of LeChatelier). This is evidence that calcium carbonate clusters of the prenucleation stage form on the basis of equilibrium thermodynamics. These clusters are thermodynamically stable and not metastable as classically considered, because an equilibrium constant of cluster formation exists, which corresponds to a minimum in Gibbs energy (Fig. 2)

Indeed, the binding of carbonate ions in the clusters can be quantitatively evaluated by constant $\mathrm{pH}$ titration. The binding of carbonate ions in the clusters (and in particles after nucleation) requires titration with dilute $\mathrm{NaOH}$ to maintain constant pH (SOM section 2.1 and fig. S4). Analyses show that calcium binding (Fig. 1B) is congruent to carbonate binding within experimental accuracy at all $\mathrm{pH}$ values investigated (SOM section 2.2 and fig. S5), that is, the formed clusters are neutral on average. The thermodynamics of cluster formation cannot be quantitatively characterized assuming the equilibrium $z \times \mathrm{Ca}^{2+}+z \times \mathrm{CO}_{3}{ }^{2-} \rightleftharpoons\left[\mathrm{CaCO}_{3}\right]_{z}$, because an excess of variables remains unknown, that is, the number of ions combined in clusters $(z)$, the equilibrium constant of cluster formation, and the clusters' $\left(\left[\mathrm{CaCO}_{3}\right]_{z, \text { aq }}\right)$ activity. Such equilibrium is valid for all concentrations of calcium and carbonate ions; thus, clusters form in the undersaturated stage (Fig. 1A) and most likely also in the presence of solid calcium carbonate, and the solubility product of calcium carbonate may account for a low concentration of clusters in the presence of solid $\mathrm{CaCO}_{3}$.

The prenucleation-stage clusters can be independently detected by means of analytical ultracentrifugation (AUC) (SOM section 2.3, fig. S6, and table S1). The clusters cannot be detected in the undersaturated stage of the experiments, while their existence is evidenced by potential

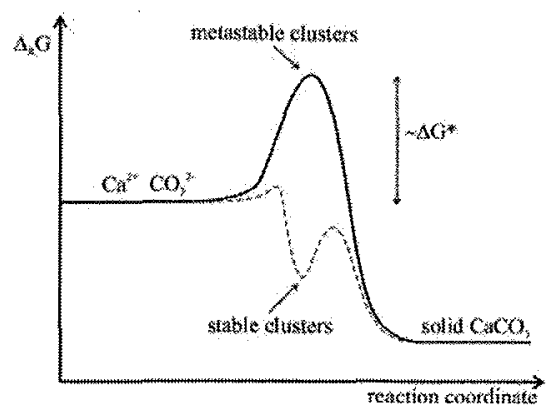

Fig. 2. Schematic illustration of the free reaction enthalpy $\Delta_{R} G$ versus the reaction coordinate. In the classical view (bold line), metastable clusters form and nucleation occurs when the critical nucleation enthalpy $\Delta G^{\star}$ is overcome. In fact, stable clusters (dashed line) are formed with an activation barrier negligible compared to thermal energy. The structure and depth of the indicated minimum remain unknown, as well as the height of the activation barrier for nucleation. 
measurements and constant $\mathrm{pH}$ titration (Fig. 1). Proximately, the cluster concentration is too low for detection by AUC in this stage. A cluster species with a hydrodynamic diameter of $\sim 2 \mathrm{~nm}$ can be detected with good statistical significance in the supersaturated stage and close to nucleation. This size corresponds to roughly 70 calcium and carbonate ions combined in single clusters on average. A second, larger cluster species (hydrodynamic diameter $\sim 4 \mathrm{~nm}$ ) can be detected, too, although with low statistical relevance. In fact, an even larger cluster species (hydrodynamic diameter -5 to $6 \mathrm{~nm}$ ) can be detected in the early postnucleation stage. The smaller cluster species cannot be detected anymore after nucleation; however, the concentration of these species may be too low for detection. These findings suggest that nucleation takes place through cluster aggregation.

The cluster equilibrium can be quantitatively characterized by means of a multiple-binding model usually applied in protein/ligand binding equilibria (26) (SOM section 2.4 and figs. S7 to $\mathrm{S} 10$ ). The derived binding parameters are $\mathrm{pH}$-dependent and relate to the binding strength in clusters. This $\mathrm{pH}$-dependent change of binding strength (fig. S11) gives a possible basis for structural preformation and the nucleation of different ACC species discussed above, which later transform into the particular crystalline poly- morph. ACC is initially nucleated as revealed by polarized light microscopy (fig. S12), and the analysis of the time development of ion products (Fig. 3) shows that, in fact, two different ACC phases are precipitated - a more stable phase (ACC I) at high binding strength in clusters $(\mathrm{pH}=9.00$ to 9.50$)$ and a less stable phase (ACC II) at low binding strength in clusters $(\mathrm{pH}=9.75$ to 10.0$)$.

WAXS (wide angle $x$-ray scattering) analysis of the crystalline particles finally formed at ambient conditions shows that pure calcite (the stable polymorph of calcium carbonate) forms at $\mathrm{pH}$-values corresponding to high binding strength in clusters and that predominantly vaterite (the least stable polymorph) and traces of calcite form at $\mathrm{pH}$-values corresponding to low binding strength in clusters (fig. S13). Both polymorphs finally form in parallel at intermediate binding strength, whereas aragonite (the intermediate stable polymorph) could not be detected. This suggests that ACC I may relate to an amorphous phase exhibiting calcitic short-range order and that ACC II may relate to an amorphous phase exhibiting vateritic short-range order. The solubilities (Fig. 3) show a discrete differentiation of the ACC phases in between $\mathrm{pH} 9.50$ and 9.75 , whereas distinct amounts of vaterite are precipitated when the ion product is still dominated by ACC I $(\mathrm{pH}=9.40)$ (fig. S13). In our opinion,

Fig. 3. Time development of the free ion product. Shown are averaged values obtained from a sample of three measurements. Because averaging is not appropriate during nucleation, the particular developments are indicated by dashed lines. We find two different ACC phases with solubitity products of $\sim 3.1 \times 10^{-8} \mathrm{M}^{2}$ (ACC 1 ) and $\sim 3.8 \times 10^{-8} \mathrm{M}^{2}$ (ACC II), corresponding to the $\mathrm{pH}$ dependency of the prenucleation cluster equilibrium. Also given are the solubilities of vaterite, aragonite, and calcite (27) (SOM section 2.5.)
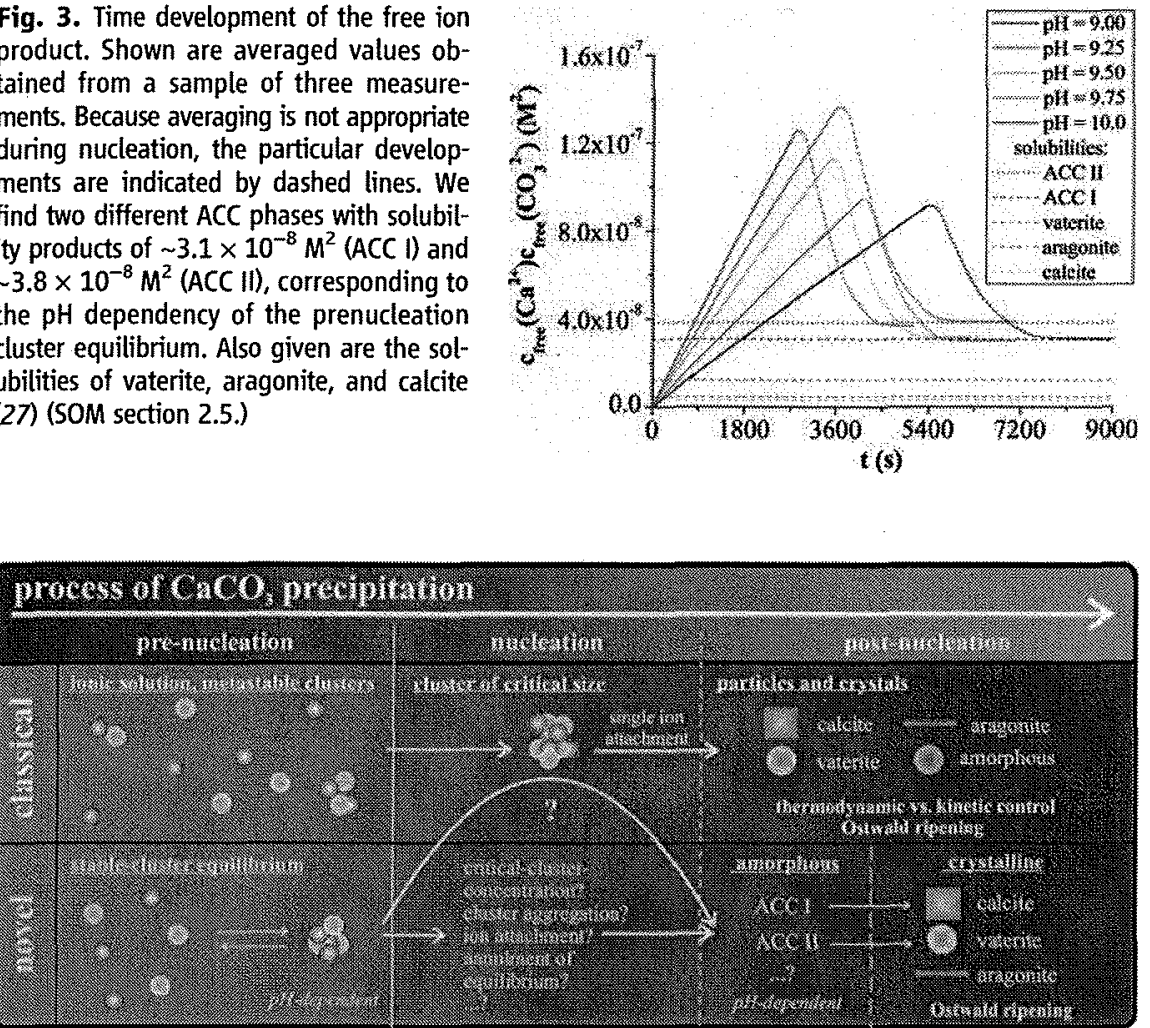

Fig. 4. Schema of the classical and novel view on precipitation (not to scale). Prenucleation-stage calcium carbonate clusters provide an early precursor species of different ACC phases giving rise to an alternative crystallization-reaction channel. both ACC phases are precipitated in parallel at intermediate binding strength, that is, the system is not yet in thermodynamic equilibrium (Gibbs' phase rule). The coexistence may not be resolved by the development of the ion product (Fig. 3) because ACC II may become crystalline fast, and the solubility of ACC I may be covered by ACC II because the solubility is dominated by the most soluble species. This is also true for minimum binding strength $(\mathrm{pH}=9.75)$, at which traces of calcite are still obtained. The correlation between binding strength in clusters and the kind of amorphous phase and polymorph finaliy formed furthermore suggests that the clusters are direct precursor species of $\mathrm{ACC}$, in which the particular structure may be also preformed. The accurate mechanism, that is, whether the nucleation of different ACC phases is under thermodynamic or kinetic control, remains unknown.

The proposed mechanism of calcium carbonate precipitation allows for early structural preformation during the prenucleation stage conveyed into the postnucleation stage (Fig. 4). The classical view, in contrast, does not facilitate such early structural preformation because ionic solutions form clusters randomly. The classical critical stage is characterized by the stochastic formation of clusters of critical size, which are thermodynamically able to grow without limit but are in fact a rare species. The growth of these clusters is then considered to take place by the addition of single ions, and the formation of different polymorphs is considered to be under thermodynamic or kinetic control. We have shown that prenucleationstage clusters form on the basis of $\mathrm{pH}$-dependent equilibrium thermodynamics (Fig. 4). The clusters show an average size of $\sim 70$ ions ( $\mathrm{pH}=9.00$ ), which is larger than expected from the classically considered, exponentially decreasing cluster size distribution. A precise cluster size distribution, however, is yet unknown. Also a precise $(\mathrm{pH}-$ dependent) cluster structure remains unknown, but it is apparent that prenucleation-stage clusters exhibit "solute character." This means that not surface tension, which is a characteristic property of phase boundaries and is classically attributed to clusters, but hydration energy taking solvent effects into account can be ascribed to clusters. The surface tension characterizing a phase interface establishes when the critical stage is reached, and amorphous $\mathrm{CaCO}_{3}$ is precipitated at first. It remains unknown whether the clusters form critical nuclei that grow classically by single-ion attachment or aggregate and then precipitate and how the precipitation of different $A C C$ phases at different $\mathrm{pH}$ values can be explained precisely, because several options exist (Fig. 4). In fact, AUC experiments provide evidence that the clusters are the nucleation-relevant species, because small cluster species cannot be detected after nucleation. In our opinion, nucleation is most probable cluster aggregation, supported by the detection of larger clusters in the early postnucleation stage and close to nucleation by means of AUC. Comparing the classical and novel view, 
it is furthermore crucial to note that a distinct part of the nucleation driving force given by the change in Gibbs energy is already released by stable cluster formation (SOM section 2.6, fig. S14, and Fig. 2).

Prenucleation-stage cluster formation on the basis of equilibrium thermodynamics can be qualitatively shown also for the biominerals calcium phosphate and calcium oxalate (SOM section 2.7 and fig. S15) and suggests a similar nucleation mechanism for these minerals. The clusterformation mechanism on the basis of equilibrium thermodynamics can be speculatively explained by entropic solvent effects. The probable release of water molecules from the hydration layer of ions caused by cluster formation may result in an increased number of degrees of freedom of the system. In classical nucleation theories, only enthalpic effects (interaction potentials) are taken into account, and entropic solvent effects are neglected. In the end, a pH-dependent change of ionic hydration layers may explain the $\mathrm{pH}$ dependency of cluster-formation thermodynamics.

\section{References and Notes}

1. R. E. Zeebe, J. C. Zachos, K. Caldeira, T. Tyrrell, Science 321. $51(2008)$
2. 1.1. De Yoreo, P. G. Vekilov, Rev, Mineral Geochem, 54 , 57 (2003).

3. M. Niederberger, H. Cölfen, Phys. Chem. Chem. Phys. 8, 3271 (2006).

4. 1. Rieger et al., Faraday Discuss. 136, 265 (2007).

5. M. Faatz, F. Gröhn, G. Wegner, Adv. Mater, 16, 996 (2004).

6. L. Addadi, S. Raz, S. Weiner, Adv. Mater. 15, 959 2003)

7. A. W. Xu, Y. R. Ma, H. Cölfen, J. Mater. Chem. 17, 415 (2007)

8. L. B. Gower, D. J. Odom, J. Cryst. Growth 210, 719 $(2000)$

9. Y. Potiti et al., Adv. Funct. Mater. 16, 1289 (2006)

10. R. S. K. Lam, J. M. Charnack, A. Lennie, F. C. Meldrum, Cryst. Eng. Comm. 9, 1226 (2007)

11. B. Hasse, H. Ehrenberg, ]. C. Marxen, W. Becker, M. Epple, Chem. Eur. J. 6, 3679 (2000).

12. C. G. Sinn, R. Dimova, M. Antonietti, Macromolecules 37 3444 (2004).

13. D. Quigley, P. M. Rodger, J. Chem. Phys. 128, 4 (2008)

14. M. Votmer, Kinetik der Phasenbildung (Steinkopff, Dresden, 1939).

15. R. Becker, W. Döring, Annalen Der Physik 24, 719 (1935)

16. A. Navrotsky, Proc, Natl. Acad. Sci. U.S.A. 101, 12096 (2004).

17. D. Horn, J. Rieger, Angew. Chem. Int. Ed. 40, 4330 (2001).

18. C. C. Perry, Biomineralization 54, 291 (2003)

19. G. Furrer, B. L. Phillips, K U Ulrich, R. Pothig W. H. Casev. Science 297, 2245 (2002)
20. W. H. Casey, I. W. Swaddle, Rev, Geophys, 41, 1008 (2003)

21. M. A. Larson, J. Garside, Chem. Eng. Sci. 41, 1285 (1986)

22. M. A. Larson, Adv. Ind. Cryst. 1991, 20 (1991).

23. D. Knezic, ]. Zaccaro, A. S. Myerson, J. Phys. Chem. $B$ 108, $10672(2004)$

24. A. F. Izmailov, A. S. Myerson, S. Arnold, J. Cryst. Growth 196, 234 (1999)

25. V. K. Lamer, R. H. Dinegar, J. Am. Chem Soc, 72,4847 (1950)

26. G. Scatchard, Ann. N. Y. Acad. Sci. 51, 660 (1949).

27. L. Brecevic, A. E. Nielsen, I. Cryst. Growth 98, 504 (1989).

28. Financial support by the German Science Foundation priority program 1117 "Principles of Biomineralization" and the Max Planck Society are gratefully acknowiedged. We thank M. Antonietti (MPI) and P. Bowen (Ecole Polytechnique Fédérale de Lausanne, Switzerland) for valuable discussions on the manuscript, $A$. Verch (MPI) for experimental assistance, and $O$. Paris and 1. Zenke (both MPI) for SAXS analysis. D.G. thanks T. Ketterer and 1. Thomas of Deutsche Metrohm for technical assistance. 